\title{
ON THE ALTERNATING PROJECTIONS THEOREM AND BIVARIATE STATIONARY STOCHASTIC PROCESSES
}

\author{
BY \\ HABIB SALEHI( $\left.{ }^{1}\right)$
}

Summary. In this paper we shall first use the theorem of von Neumann on alternating projections to obtain an algorithm for finding the projection of an element $x$ in a Hilbert space $\mathscr{H}$ onto the subspace spanned by $\mathscr{H}$-valued orthogonally scattered measures $\xi_{1}$ and $\xi_{2}$. We then specialize this algorithm to the case that $\xi_{1}$ and $\xi_{2}$ are the canonical measures of the components of a bivariate stationary stochastic process (SP), and thereby get an algorithm for finding the best linear predictor in the time domain.

1. Preliminary results. In this section we state some results which are used in later sections.

The following theorem is due to J. von Neumann (cf. [7, p. 55]).

Theorem 1.1 (Alternating Projections). Let $P_{1}, P_{2}$, and $T$ be projection operators on a Hilbert space $\mathscr{H}$ onto the subspaces $\mathscr{M}_{1}, \mathscr{M}_{2}$, and $\mathscr{M}_{1} \cap \mathscr{M}_{2}$. If $T_{n}$ is the nth term of either of the sequences

$$
\begin{aligned}
& P_{1}, P_{2} P_{1}, P_{1} P_{2} P_{1}, P_{2} P_{1} P_{2} P_{1}, \ldots, \\
& P_{2}, P_{1} P_{2}, P_{2} P_{1} P_{2}, P_{1} P_{2} P_{1} P_{2}, \ldots,
\end{aligned}
$$

then $T_{n} \rightarrow T$ strongly $\left({ }^{2}\right)$, as $n \rightarrow \infty$.

This at once yields the following corollary, which we need later.

COROLlaRY 1.2. With the notation of 1.1 , if $P$ is the projection operator on $\mathscr{H}$ onto the subspace $\mathfrak{S}\left(\mathscr{M}_{1}+\mathscr{M}_{2}\right)$ which is spanned by $\mathscr{M}_{1}$ and $\mathscr{M}_{2}$, then

$$
P=P_{1}+P_{2}-P_{1} P_{2}-P_{2} P_{1}+P_{1} P_{2} P_{1}+P_{2} P_{1} P_{2}-\cdots
$$

the convergence being in the strong sense.

The next theorem is due to A. S. Besicovitch (cf. [1, p. 9]).

THEOREM 1.3. Let (i) $\mu$ be a bounded, countably additive (c.a.), nonnegative measure defined on the family $\mathscr{B}$ of Borel subsets of the real line $R$.

(ii) $\nu$ be a c.a., complex-valued measure on $\mathscr{B}$.

(iii) $D(\lambda, h)=\{\nu(\lambda-h, \lambda+h) / \mu(\lambda-h, \lambda+h)\} \chi_{\sigma(\mu)}(\lambda)$, where $\sigma(\mu)$ is the spectrum of $\mu$, i.e., $\sigma(\mu)=\{\lambda$ : for each $h>0, \mu(\lambda-h, \lambda+h)>0\}$. Then (a) the limit $D(\lambda, h)$, as $h \rightarrow 0$, exists (finite) almost everywhere with respect to $\mu$ (a.e. $\mu$ ).

Received by the editors June 15, 1966.

(1) Publication supported by Public Health Service Research Grant No. NIH-GM-1313801 from the National Institute of General Medical Sciences.

(2) I.e., for each $x$ in $\mathscr{H},\left|T_{n} x-P x\right| \rightarrow 0$, as $n \rightarrow \infty,|\quad|$ being the norm in $\mathscr{H}$. 
(b) If $\nu$ is absolutely continuous (a.c.) with respect to $\mu$ and if the Radon-Nikodym derivative of $\nu$ with respect to $\mu$ is $d \nu / d \mu$, then

$$
(d \nu / d \mu)(\cdot)=\lim D(\cdot, h) \text {, as } h \rightarrow 0 \text {, a.e. } \mu .
$$

We shall denote $\lim D(\cdot, h)$, as $h \rightarrow 0$, by $(D \nu / D \mu)(\cdot)$ and shall call it the Besicovitch derivative of $\nu$ with respect to $\mu$.

The following is an immediate consequence of 1.3.

Corollary 1.4. Let (i) $M_{i}(i=1,2)$ be a $\sigma$-finite, c.a., nonnegative measure on the family $\mathscr{B}$ of Borel subsets of the real line $R$.

(ii) $\mathscr{B}_{i}=\left\{B: B \in \mathscr{B}_{i}\right.$ and $\left.M_{i}(B)<\infty\right\}, M_{12}$ the product measure generated by $M_{1}$ and $M_{2}$, and $\mathscr{B}_{12}=\left\{B: B\right.$ is a Borel subset of the complex plane $R^{2}$ and $\left.M_{12}(B)<\infty\right\}$.

(iii) $\mu$ be a c.a. measure on the ring $\mathscr{B}_{12}$ which is a.c. with respect to $M_{12}$.

(iv) $d \mu / d M_{12}$ be any version of the Radon-Nikodym derivative of $\mu$ with respect to $M_{12}$, which is defined throughout $R^{2}$.

(v) For each $B \in \mathscr{B}_{1}$ and each $t \in R$,

$$
p(B, t)=\int_{B}\left(d \mu / d M_{12}\right)(s, t) M_{1}(d s) .
$$

Then (a) for each $B \in \mathscr{B}_{1}, \mu_{B}(\cdot)=\mu(B \times \cdot)$ is a c.a. measure on $\mathscr{B}_{2}$ which is a.c. with respect to $M_{2}$.

(b) For each $B \in \mathscr{B}_{1}, p(B, \cdot)$ is a version of $d \mu_{B} / d M_{2}$, the domain of which is $R$.

(c) For each $t \in R, p(\cdot, t)$ is a c.a. measure on $\mathscr{B}_{1}$, which is a.c. with respect to $M_{1}$.

(d) Analogous results to (a), (b), and (c) hold for $\nu_{B}$, where for each $B \in \mathscr{B}_{2}$, $\nu_{B}(\cdot)=\mu(\cdot \times B)$.

2. Orthogonally scattered, $\mathscr{H}$-valued measures. We first state a few results concerning measures whose values are orthogonally scattered in a given Hilbert space. These are special versions of more general results contained in [9], [10], and [11].

Definition 2.1. Let (i) $M$ be a $\sigma$-finite, c.a., nonnegative measure on the family $\mathscr{B}$ of Borel subsets of the real line $R$.

(ii) $\mathscr{B}_{0}=\{B: B \in \mathscr{B}$ and $M(B)<\infty\}$.

Then a function $\xi$ on $\mathscr{B}_{0}$ into a given Hilbert space $\mathscr{H}$ such that for each pair of $B, C \in \mathscr{B}_{0}$,

$$
(\xi(B), \xi(C))=M(B \cap C)
$$

is called an orthogonally scattered (o.s.) $\mathscr{H}$-valued measure over $(R, \mathscr{B})$. $M$ is called the spectral measure associated with $\xi$. It follows immediately that $(\xi(B), \xi(C))=0$ if $B$ and $C$ are disjoint sets in $\mathscr{B}_{0}$, and $\xi\left(\bigcup_{k} B_{k}\right)=\sum_{k} \xi\left(B_{k}\right)$ if $B_{k}$ 's are disjoint sets in $\mathscr{B}_{0}$ whose union $\bigcup_{k} B_{k}$ is in $\mathscr{B}_{0}$.

There is a well-established theory of integration with respect to an o.s. $\mathscr{H}$-valued measure $\xi$ whose associated spectral measure is $\sigma$-finite (cf. [10] and [11]). We will briefly state the main result. 
THEOREM 2.2. If $\mathscr{S}$ is the set of all stochastic integrals $\int_{R} \phi d \xi$ then

(a) $\int_{R} \phi d \xi$ exists iff $\int_{R}|\phi|^{2} d M<\infty$.

(b) $\int_{R}(a \phi+b \psi) d \xi=a \int_{R} \phi d \xi+b \int_{R} \psi d \xi$, where $a$ and $b$ are complex numbers.

(c) $\mathscr{S}=$ the cyclic subspace spanned by $\xi$, i.e.,

$$
\mathscr{S}=\mathfrak{S}\left\{\xi(B) ; B \in \mathscr{B}_{0}\right\} .
$$

(d) $\left(\int_{R} \phi d \xi, \int_{R} \psi d \xi\right)=\int_{R} \phi \bar{\psi} d M$.

(e) The correspondence $\phi \rightarrow \int_{R} \phi d \xi$ is an isomorphism from $L_{2}(R, \mathscr{B}, M)$ onto $\mathscr{S}$ such that (a) and (b) hold, and therefore

$$
\left|\int_{R} \phi d \xi\right|^{2}=\int_{R}|\phi|^{2} d M
$$

Let $x$ be in $\mathscr{H}$ and $\{\xi(m)\}$ be an orthonormal basis for a subspace $\mathscr{M}$ of $\mathscr{H}$. Then $\sum_{m}|(x, \xi(m))|^{2}<\infty$ and the orthogonal projection of $x$ onto $\mathscr{M}$ is given by $\sum_{m}(x, \xi(m)) \xi(m)$. The following theorem generalizes this result.

TheOREM 2.3. Let (i) $x \in \mathscr{H}$ and $\xi$ be an o.s. $\mathscr{H}$-valued measure over $(R, \mathscr{B})$ whose associated spectral measure $M$ is $\sigma$-finite.

(ii) $P$ be the projection operator on $\mathscr{H}$ onto the cyclic subspace $\mathscr{M}$ spanned by the measure $\xi$, i.e., $\mathscr{M}=\subseteq\left\{\xi(B): B \in \mathscr{B}_{0}\right\}$, where $\mathscr{B}_{0}=\{B \in \mathscr{B} \& M(B)<\infty\}$.

Then (a) the complex-valued functions $(x, \xi)$ and $(\xi, x)$ defined by $(x, \xi)(\cdot)$ $=(x, \xi(\cdot))$ and $(\xi, x)(\cdot)=(\xi(\cdot), x)$ are c.a. measures over $(R, \mathscr{B})$ which are a.c. with respect to $M$.

(b) $(x, \xi)=(\xi, x)^{*}$ and $d(x, \xi) / d M=\{d(\xi, x) / d M\}^{*}$.

(c) $d(x, \xi) / d M$ and $d(\xi, x) / d M \in L_{2}(R, \mathscr{B}, M)$, and

$$
P x=\int_{R}\{d(x, \xi) / d M\} d \xi .
$$

Proof. Since the proof of (a) and (b) is trivial, we proceed to give the proof of (c). By 2.2(c), there exists a complex-valued function $\phi$ such that

$$
P x=\int_{R} \phi d \xi \quad \& \quad \phi \in L_{2}(R, \mathscr{B}, M) .
$$

Now by (1) and 2.2(d), for each $B \in \mathscr{B}_{0}$

$$
\begin{aligned}
(x, \xi)(B) & =(x, \xi(B))=(P x, \xi(B)) \\
& =\left(\int_{R} \phi d \xi, \int_{R} \chi_{B} d \xi\right)=\int_{B} \phi d M .
\end{aligned}
$$

It immediately follows from the Besicovitch Theorem 1.3 that

$$
d(x, \xi) / d M=\phi \text { a.e. } M \text {. }
$$

By (b), (1), and (2) the result follows. (Q.E.D.) 
COROLlaRy 2.4. Let (i) $\xi$ be an o.s. $\mathscr{H}$-valued measure over $(R, \mathscr{B})$ whose associated spectral measure $M$ is $\sigma$-finite.

(ii) $\phi \in L_{2}(R, \mathscr{B}, M)$.

Then (a) for each $x \in \mathscr{H}$, the complex-valued function $(\xi, x)$ defined in 2.3 is a c.a. measure over $(R, \mathscr{B})$ which is a.c. with respect to $M$.

(b) $\phi \in L_{1}[R, \mathscr{B},(\xi, x)]$, and

$$
\left(\int_{R} \phi(\omega) \xi(d \omega), x\right)=\int_{R} \phi(\omega)(\xi, x)(d \omega) .
$$

Proof. The proof of (a) follows from 2.3(a). Let $P$ denote the projection operator on $\mathscr{H}$ onto the cyclic subspace $\mathscr{M}$ spanned by the measure $\xi$. Since $\phi \in L_{2}(R, \mathscr{B}, M)$ and by $2.3(\mathrm{c}), d(\xi, x) / d M \in L_{2}(R, \mathscr{B}, M)$, therefore $\phi d(x, \xi) / d M \in L_{1}(R, \mathscr{B}, M)$ or equivalently $\phi \in L_{1}[R, \mathscr{B},(\xi, x)]$. To prove the second part of (c), we observe that by $2.2(\mathrm{c}), \int_{R} \phi d \xi \in \mathscr{M}$ and therefore

$$
\left(\int_{R} \phi d \xi, x\right)=\left(\int_{R} \phi d \xi, P x\right)
$$

Now by 2.2(d), 2.3(b), and 2.3(c),

$$
\begin{aligned}
\left(\int_{R} \phi d \xi, P x\right) & =\left(\int_{R} \phi d \xi, \int_{R} \frac{d(x, \xi)}{d M} d \xi\right)=\int_{R} \phi(\omega) \frac{(d(x, \xi))^{*}}{d M}(\omega) M(d \omega) \\
& =\int_{R} \phi(\omega) \frac{d(\xi, x)}{d M}(\omega) M(d \omega)=\int_{R} \phi(\omega)(\xi, x)(d \omega) .
\end{aligned}
$$

By (1) and (2) we get the result. (Q.E.D.)

3. The alternating projections theorem and $\mathscr{H}$-valued measures. Let $\left\{\xi_{1}(m)\right\}$ and $\left\{\xi_{2}(m)\right\}$ be orthonormal bases for the subspaces $\mathscr{M}_{1}$ and $\mathscr{M}_{2}$ of a given Hilbert space $\mathscr{H}$. Let $P_{1}, P_{2}$, and $P$ be projection operators on $\mathscr{H}$ onto the subspaces $\mathscr{M}_{1}, \mathscr{M}_{2}$, and $S\left(\mathscr{M}_{1}+\mathscr{M}_{2}\right)$. From 1.2 it follows that for each $x$ in $\mathscr{H}$

$$
\begin{aligned}
P x= & \sum_{m}\left(x, \xi_{1}(m)\right) \xi_{1}(m)+\sum_{m}\left(x, \xi_{2}(m)\right) \xi_{2}(m) \\
& -\sum_{n}\left\{\sum_{m}\left(x, \xi_{2}(m)\right)\left(\xi_{2}(m), \xi_{1}(n)\right)\right\} \xi_{1}(n) \\
& -\sum_{n}\left\{\sum_{m}\left(x, \xi_{1}(m)\right)\left(\xi_{1}(m), \xi_{2}(n)\right)\right\} \xi_{2}(n) \\
& +\cdots .
\end{aligned}
$$

Now an orthonormal basis is just an o.s. $\mathscr{H}$-valued measure over $(R, \mathscr{B})$ which is concentrated on the set of integers. In many situations it is more natural to think of a subspace $\mathscr{M}$ as being spanned by such a measure than by some basis, and to represent a vector in $\mathscr{H}$ by an integral rather than by a sum. (This happens, for instance, in the theory of the Fourier integral.) 
We shall generalize equation (A) to the case in which $\mathscr{M}_{1}$ and $\mathscr{M}_{2}$ are spanned by such measures $\xi_{1}$ and $\xi_{2}$. We shall show that under certain conditions we get instead of (A):

$$
\begin{aligned}
P x= & \int_{R} \frac{d\left(x, \xi_{1}\right)}{d M_{1}}(s) \xi_{1}(d s)+\int_{R} \frac{d\left(x, \xi_{2}\right)}{d M_{2}}(s) \xi_{2}(d s) \\
& -\int_{R}\left\{\int_{R} \frac{d\left(x, \xi_{2}\right)}{d M_{2}}(s) \frac{d\left(\xi_{2}, \xi_{1}\right)}{d M_{21}}(s, t) M_{2}(d s)\right\} \xi_{1}(d t) \\
& -\int_{R}\left\{\int_{R} \frac{d\left(x, \xi_{1}\right)}{d M_{1}}(s) \frac{d\left(\xi_{1}, \xi_{2}\right)}{d M_{12}}(s, t) M_{1}(d s)\right\} \xi_{2}(d t)+\cdots
\end{aligned}
$$

where $M_{12}, M_{21}$ are the product measures $M_{1} \times M_{2}, M_{2} \times M_{1}$, and $d(\quad) / d$ denotes the Radon-Nikodym derivative.

Notation 3.1. $\mathscr{B}$ will denote the family of Borel subsets of the real line $R$. $\mathscr{H}$ will denote a fixed Hilbert space. $\xi_{i}(i=1,2)$ will denote an o.s. $\mathscr{H}$-valued measure over $(R, \mathscr{B})$ whose associated spectral measure $M_{i}(i=1,2)$ is $\sigma$-finite. We set

$\mathscr{B}_{i}=\left\{B: B \in \mathscr{B} \quad \& \quad M_{i}(B)<\infty\right\}, 1 \leqq i \leqq 2$,

$\mathscr{B} \times \mathscr{B}=$ the family of Borel subsets of the complex plane $R^{2}$.

$\mathscr{B}_{i} \times \mathscr{B}_{j}=\left\{B \times C: B \in \mathscr{B}_{i} \& C \in \mathscr{B}_{j}\right\}, 1 \leqq i, j \leqq 2$,

$\mathscr{R}_{i j}=$ the ring generated by $\mathscr{B}_{i} \times \mathscr{B}_{j}, 1 \leqq i, j \leqq 2$,

$M_{i j}=$ the product measure $M_{i} \times M_{j}$ on $\mathscr{B} \times \mathscr{B}, 1 \leqq i, j \leqq 2$,

$\mathscr{B}_{i j}=\left\{B: B \in \mathscr{B} \times \mathscr{B} \quad \& \quad M_{i j}(B)<\infty\right\}, 1 \leqq i, j \leqq 2$.

To be able to carry out our work we need to study the complex-valued measure $\left(\xi_{i}, \xi_{j}\right)$ which is generated by $\xi_{i}$ and $\xi_{j}$. Our definition of this measure will be given in several steps.

Definition 3.2. We define $\left(\xi_{i}, \xi_{j}\right)$ on $\mathscr{B}_{i} \times \mathscr{B}_{j}$ by:

$$
\left(\xi_{i}, \xi_{j}\right)(B \times C)=\left(\xi_{i}(B), \xi_{j}(C)\right) .
$$

A usual argument may be used to prove the following lemma.

LEMMA 3.2. Let $\left\{B_{k}\right\}_{k=1}^{m}$ and $\left\{C_{l}\right\}_{l=1}^{n}$ be finite sequences of sets in $\mathscr{B}_{i} \times \mathscr{B}_{j}$ such that $\bigcup_{k=1}^{m} B_{k}=\bigcup_{l=1}^{n} C_{l}$. Then

$$
\sum_{k=1}^{m}\left(\xi_{i}, \xi_{j}\right)\left(B_{k}\right)=\sum_{l=1}^{n}\left(\xi_{i}, \xi_{j}\right)\left(C_{l}\right)
$$

Since every $B \in \mathscr{R}_{i j}$ is a finite, disjoint union of sets in $\mathscr{B}_{i} \times \mathscr{B}_{j}$ (cf. [3, p. 139]), from 3.2 it follows that $\left(\xi_{i}, \xi_{j}\right)$ can be defined on $\mathscr{R}_{i j}$ in the following way.

Definition 3.3. Let $B \in \mathscr{R}_{i j}$. Then we define

$$
\left(\xi_{i}, \xi_{j}\right)(B)=\sum_{k=1}^{n}\left(\xi_{i}, \xi_{j}\right)\left(B_{k}\right),
$$

where the $B_{k}$ 's are any finite sequence of disjoint sets in $\mathscr{B}_{i} \times \mathscr{B}_{j}$ such that $B=\bigcup_{k=1}^{n} B_{k}$. 
The next lemma whose proof is easily seen shows that $\left(\xi_{i}, \xi_{j}\right)$ is finitely additive on $\mathscr{R}_{i j}$.

LEMMA 3.4. (a) $\left(\xi_{i}, \xi_{j}\right)$ is finitely additive on $\mathscr{R}_{i j}$ and is a.c. with respect to $M_{i j}$ on $\mathscr{R}_{i j}$.

(b) $\left(\xi_{i}, \xi_{j}\right)$ is the unique finitely additive extension of the measure defined in 3.2 on $\mathscr{B}_{i} \times \mathscr{B}_{j}$ to $\mathscr{R}_{i j}$.

To proceed further, some restriction has to be imposed on $\xi_{1}$ and $\xi_{2}$.

Assumption 3.5. There exist functions $\varphi_{12}$ and $\varphi_{21}$ on $R^{2}$ such that

(1) $\varphi_{i j}(\cdot, t) \in L_{2}\left(R, \mathscr{B}, M_{i}\right)$ a.e. $t\left(M_{j}\right)$,

$M_{j}$ - ess. lub.teR $\left|\varphi_{i j}(\cdot, t)\right|_{2, M_{i}}<\infty\left({ }^{3}\right)$.

(2) For each $B \in \mathscr{B}_{i j}, \chi_{B} \varphi_{i j} \in L_{1}\left(R^{2}, \mathscr{B} \times \mathscr{B}, M_{i j}\right)$.

(3) For each $B \times C \in \mathscr{B}_{i} \times \mathscr{B}_{\text {j }}$

$$
\left(\xi_{i}(B), \xi_{j}(C)\right)=\iint_{B \times C} \varphi_{i j}(s, t) M_{i j}(d(s, t)) .
$$

We will see later (cf. 4.5) that in many situations Assumption 3.5 is satisfied.

LEMMA 3.6. Let (i) $\xi_{1}$ and $\xi_{2}$ be o.s. $\mathscr{H}$-valued measures over $(R, \mathscr{B})$ satisfying Assumption 3.5. Then (a) the measure $\left(\xi_{i}, \xi_{j}\right)$ on $R_{i j}$, introduced in 3.3 , has a c.a. extension $\mu_{i j}$ to $\mathscr{B}_{i j}$ such that for each $B \in \mathscr{B}_{i j}$,

$$
\mu_{i j}(B)=\iint_{B} \varphi_{i j}(s, t) M_{i j}(d(s, t)) .
$$

(b) $\mu_{i j}$ is a.c. with respect to $M_{i j}$ and $d \mu_{i j} / d M_{i j}=\varphi_{i j}$ a.e. $M_{i j}$.

Definition 3.7. We define $\left(\xi_{i}, \xi_{j}\right)$ on $\mathscr{B}_{i j}$ to be the $\mu_{i j}$ of the last lemma.

In the next theorem we state some consequences which occur when we have a c.a. measure $\left(\xi_{i}, \xi_{j}\right)$ as above. The proof is immediate from 1.4.

THEOREM 3.8. With the notation of 3.1, if $\left(\xi_{i}, \xi_{j}\right)$ is any c.a. measure on $\mathscr{B}_{i j}$ which is a.c. with respect to $M_{i j}$ such that for each $B \times C \in \mathscr{B}_{i} \times \mathscr{B}_{j},\left(\xi_{i}, \xi_{j}\right)(B \times C)$ $=\left(\xi_{i}(B), \xi_{j}(C)\right)$. Then (a) for each $B \in \mathscr{B}_{j},\left(\xi_{i}, \xi_{j}(B)\right)$ is a c.a. measure on $\mathscr{B}_{i}$ which is a.c. with respect to $M_{i}$.

(b) For each $B \in \mathscr{B}_{j}, \int_{B}\left\{d\left(\xi_{i}, \xi_{j}\right) / d M_{i j}\right\}(\cdot, t) M_{j}(d t)$ is a version of $d\left(\xi_{i}, \xi_{j}(B)\right) / d M_{i}$.

(c) For almost all $s\left(M_{i}\right),\left\{d\left(\xi_{i}, \xi_{j}(\cdot)\right) / d M_{i}\right\}(s)$ is a c.a. measure on $\mathscr{B}_{j}$ which is a.c. with respect to $M_{j}$.

(d) For each $B \in \mathscr{B}_{i}$ similar results hold for the measure $\left(\xi_{i}(B), \xi_{j}\right)$.

We shall now state the main theorem of this section.

(3) By definition $\left|\varphi_{i j}(\cdot, t)\right|_{2, M_{i}}=\left[\int_{R}\left|\varphi_{i j}(s, t)\right|^{2} M_{i}(d s)\right]^{1 / 2}$. 
THEOREM 3.9. With the notation of 3.1 , if $\xi_{i}(i=1,2)$ are o.s. $\mathscr{H}$-valued measures over $(R, \mathscr{B})$ satisfying Assumption 3.5 , and if $\left(\xi_{i}, \xi_{j}\right)$ is the c.a. measure on $\mathscr{B}_{i j}$ given by 3.7, then for each $x$ in $\mathscr{H}$

$$
\begin{gathered}
d\left(x, \xi_{i}\right) / d M_{i} \in L_{2}\left(R, \mathscr{B}, M_{i}\right), \\
\frac{d\left(x, \xi_{i}\right)}{d M_{i}}(\cdot) \frac{d\left(\xi_{i}, \xi_{j}\right)}{d M_{i j}}(\cdot, t) \in L_{1}\left(R, \mathscr{B}, M_{i}\right) \text { a.e. } t\left(M_{j}\right), \\
\int_{R} \frac{d\left(x, \xi_{i}\right)}{d M_{i}}(s) \frac{d\left(\xi_{i}, \xi_{j}\right)}{d M_{i j}}(s, \cdot) M_{i}(d s) \in L_{2}\left(R, \mathscr{B}, M_{j}\right),
\end{gathered}
$$$$
\left\{\int_{R} \frac{d\left(\dot{x}, \xi_{i}\right)}{d M_{i}}(s) \frac{d\left(\xi_{i}, \xi_{j}\right)}{d M_{i j}}(s, \cdot) M_{i}(d s)\right\} \frac{d\left(\xi_{j}, \xi_{i}\right)}{d M_{j i}}(\cdot, u) \in L_{1}\left(R, \mathscr{B}, M_{j}\right) \text { a.e. } u\left(M_{i}\right),
$$$$
\int_{R}\left\{\int_{R} \frac{d\left(x, \xi_{i}\right)}{d M_{i}}(s) \frac{d\left(\xi_{i}, \xi_{j}\right)}{d M_{i j}}(s, t) M_{i}(d s)\right\} \frac{d\left(\xi_{j}, \xi_{i}\right)}{d M_{j i}}(t, \cdot) M_{j}(d t) \in L_{2}\left(R, \mathscr{B}, M_{i}\right), \ldots
$$

(b) If $P_{i}(i=1,2)$ is the projection operator onto the cyclic subspaces $\mathscr{M}_{i}(i=1,2)$ which is spanned by $\xi_{i}(i=1,2)$, then

$$
\begin{aligned}
P_{i} x & =\int_{R} \frac{d\left(x, \xi_{i}\right)}{d M_{i}}(s) \xi_{i}(d s), \\
P_{j} P_{i} x & =\int_{R}\left\{\int_{R} \frac{d\left(x, \xi_{i}\right)}{d M_{i}}(s) \frac{d\left(\xi_{i}, \xi_{j}\right)}{d M_{i j}}(s, t) M_{i}(d s)\right\} \xi_{j}(d t), \\
P_{i} P_{j} P_{i} x & =\int_{R}\left\{\int_{R}\left\{\int_{R} \frac{d\left(x, \xi_{i}\right)}{d M_{i}}(s) \frac{d\left(\xi_{i}, \xi_{j}\right)}{d M_{i j}}(s, t)\right\} \frac{d\left(\xi_{j}, \xi_{i}\right)}{d M_{j i}}(t, u) M_{j}(d u)\right\} \xi_{i}(d u), \ldots
\end{aligned}
$$

Proof. For simplicity let $i=1$ and $j=2$. By 2.3,

$$
\frac{d\left(x, \xi_{1}\right)}{d M_{1}} \in L_{2}\left(R, \mathscr{B}, M_{1}\right) \text { and } P_{1} x=\int_{R} \frac{d\left(x, \xi_{1}\right)}{d M_{1}}(s) \xi_{1}(d s) .
$$

Replacing $x$ by $P_{1} x$ and $\xi_{1}$ by $\xi_{2}$ in (1) we get

$$
\frac{d\left(P_{1} x, \xi_{2}\right)}{d M_{2}} \in L_{2}\left(R, \mathscr{B}, M_{2}\right) \quad \text { and } \quad P_{2} P_{1} x=\int_{R} \frac{d\left(P_{1} x, \xi_{2}\right)}{d M_{2}}(t) \xi_{2}(d t) .
$$

Let $B \in \mathscr{B}_{2}$. Then we have

$$
\begin{array}{rlrl}
\left(P_{1} x, \xi_{2}(B)\right) & =\left(\int_{R} \frac{d\left(x, \xi_{1}\right)}{d M_{1}}(s) \xi_{1}(d s), \xi_{2}(B)\right) & & \text { by (1) } \\
& =\int_{R} \frac{d\left(x, \xi_{1}\right)}{d M_{1}}(s)\left(\xi_{1}(d s), \xi_{2}(B)\right) & & \text { by } 2.4 \\
& =\int_{R} \frac{d\left(x, \xi_{1}\right)}{d M_{1}}(s) \frac{d\left(\xi_{1}, \xi_{2}(B)\right)}{d M_{1}}(s) M_{1}(d s) & & \text { by 3.8(a) } \\
& =\int_{R} \frac{d\left(x, \xi_{1}\right)}{d M_{1}}(s)\left\{\int_{B} \frac{d\left(\xi_{1}, \xi_{2}\right)}{d M_{12}}(s, t) M_{2}(d t)\right\} M_{1}(d s) & & \text { by 3.8(b) } \\
& =\int_{B}\left\{\int_{R} \frac{d\left(x, \xi_{1}\right)}{d M_{1}}(s) \frac{d\left(\xi_{1}, \xi_{2}\right)}{d M_{12}}(s, t) M_{1}(d s)\right\} M_{2}(d t), &
\end{array}
$$

where in the last step, the change of order of integration can be justified as follows: 
By the Schwarz inequality, we have

$$
\begin{aligned}
\int_{R}\left|\frac{d\left(x, \xi_{1}\right)}{d M_{1}}(s)\right|\left|\frac{d\left(\xi_{1}, \xi_{2}\right)}{d M_{12}}(s, t)\right| M_{1}(d s) \\
\qquad\left[\int_{R}\left|\frac{d\left(x, \xi_{1}\right)}{d M_{1}}(s)\right|^{2} M_{1}(d s)\right]^{1 / 2} \times\left[\int_{R}\left|\frac{d\left(\xi_{1}, \xi_{2}\right)}{d M_{12}}(s, t)\right|^{2} M_{1}(d s)\right]^{1 / 2} \leqq C K
\end{aligned}
$$

where by (1), $C=\left[\int_{R}\left|\left(d\left(x, \xi_{1}\right) / d M_{1}\right)(s)\right|^{2} M_{1}(d s)\right]^{1 / 2}<\infty$, and by our hypothesis and Assumption 3.5, $K=M_{2}$ - ess. lub.teR $\left|\left(d\left(\xi_{1}, \xi_{2}\right) / d M_{12}\right)(\cdot, t)\right|_{2, M_{1}}<\infty$. Then

$$
\int_{B}\left\{\int_{R}\left|\frac{d\left(x, \xi_{1}\right)}{d M_{1}}(s)\right|\left|\frac{d\left(\xi_{1}, \xi_{2}\right)}{d M_{12}}(s, t)\right| M_{2}(d t)\right\} \leqq C K M_{2}(B)<\infty .
$$

Hence Fubini's Theorem (cf. [3, p. 148]) can be applied.

Since $M_{2}$ is $\sigma$-finite, there exist countably many disjoint sets $N_{i}$ such that $\sigma_{M_{2}}=\bigcup N_{i}$, where $\sigma_{M_{2}}$ is the spectrum of $M_{2}$ (cf. 1.3(iii)), and $M_{2}\left(N_{i}\right)<\infty$. If $t \in N_{i}$, taking in (3) $B=N_{i} \cap \Delta_{t, h}$, where $\Delta_{t, h}=\{s: t-h<s<t+h\}$ we get

$$
\left(P_{1} x, \xi_{2}\left(N_{i} \cap \Delta_{t, h}\right)\right)=\int_{N_{i} \cap \Delta_{t, h}}\left\{\int_{R} \frac{d\left(x, \xi_{1}\right)}{d M_{1}}(s) \frac{d\left(\xi_{1}, \xi_{2}\right)}{d M_{12}}(s, u) M_{1}(d s)\right\} M_{2}(d u) .
$$

Hence by the Besicovitch Theorem 1.3

$$
\begin{aligned}
\frac{d\left(P_{1} x, \xi_{2}\right)}{d M_{2}}(t) & =\lim \frac{\left(P_{1} x, \xi_{2}\left(N_{i} \cap \Delta_{t, h}\right)\right)}{M_{2}\left(N_{i} \cap \Delta_{t, h}\right)}, \text { as } h \rightarrow 0 \\
& =\int_{R} \frac{d\left(x, \xi_{1}\right)}{d M_{1}}(s) \frac{d\left(\xi_{1}, \xi_{2}\right)}{d M_{12}}(s, t) M_{1}(d s) \text { a.e. } t \in N_{i}\left(M_{2}\right) .
\end{aligned}
$$

Since $\left\{N_{i}\right\}$ is a countable collection, and $M_{2}\left(R-\sigma_{M_{2}}\right)=0$, (4) holds a.e. $t\left(M_{2}\right)$. By (2), we see that the function on the right-hand side of (4) is in $L_{2}\left(R, \mathscr{B}, M_{2}\right)$ and that

$$
P_{2} P_{1} x=\int_{R}\left\{\int_{R} \frac{d\left(x, \xi_{1}\right)}{d M_{1}}(s) \frac{d\left(\xi_{1}, \xi_{2}\right)}{d M_{12}}(s, t) M_{1}(d s)\right\} \xi_{2}(d t)
$$

This completes the proof of the first relation in (a) and the first two relations in (b).

Tó obtain the expression for $P_{2} P_{1} x$ given by (b), we essentially made use of the fact that

$$
\begin{gathered}
M_{2} \text { - ess. lub. }\left|\frac{d\left(\xi_{1}, \xi_{2}\right)}{d M_{12}}(\cdot, t)\right|_{2, M_{1}}<\infty . \\
\frac{d\left(x, \xi_{1}\right)}{d M_{1}}(\cdot) \in L_{2}\left(R, \mathscr{B}, M_{1}\right) \\
P_{1} x=\int_{R} \frac{d\left(x, \xi_{1}\right)}{d M_{1}} d \xi_{1} .
\end{gathered}
$$


Now since

$$
\begin{gathered}
M_{1}-\underset{t \in R}{\text { ess. lub. }}\left|\frac{d\left(\xi_{2}, \xi_{1}\right)}{d M_{21}}(\cdot, u)\right|_{2, M_{2}}<\infty, \\
\phi(\cdot)=\int_{R} \frac{d\left(x, \xi_{1}\right)}{d M_{1}}(s) \frac{d\left(\xi_{1}, \xi_{2}\right)}{d M_{12}}(s ; \cdot) M_{1}(d s) \in L_{2}\left(R, \mathscr{B}, M_{2}\right), \\
P_{2} P_{1} x=\int_{R} \phi d \xi_{2},
\end{gathered}
$$

therefore in exactly the same way as before we can obtain the expression for $P_{1} P_{2} P_{1} x$ given in (b). The rest of the proof may be completed by induction. (Q.E.D.)

The following corollary is an immediate consequence of this theorem and 1.2.

Corollary 3.10. With the notation of 3.1 , if $\xi_{i}(i=1,2)$ are o.s. $\mathscr{H}$-valued measures satisfying Assumption 3.5 and if $P_{i}(i=1,2)$ and $P$ are the projection operators on $\mathscr{H}$ onto the cyclic subspaces $\mathscr{M}_{i}=\subseteq\left\{\xi_{i}(B), B \in \mathscr{B}_{i}\right\}(i=1,2)$ and $\subseteq\left\{\mathscr{M}_{1}+\mathscr{M}_{2}\right\}$, then for each $x \in \mathscr{H}$,

$$
\begin{aligned}
P x= & \int_{R} \frac{d\left(x, \xi_{1}\right)}{d M_{1}}(s) \xi_{1}(d s)+\int_{R} \frac{d\left(x, \xi_{2}\right)}{d M_{2}}(s) \xi_{2}(d s) \\
& -\int_{R}\left\{\int_{R} \frac{d\left(x, \xi_{2}\right)}{d M_{2}}(s) \frac{d\left(\xi_{2}, \xi_{1}\right)}{d M_{21}}(s, t) M_{2}(d s)\right\} \xi_{1}(d t) \\
& -\int_{R}\left\{\int_{R} \frac{d\left(x, \xi_{1}\right)}{d M_{1}}(s) \frac{d\left(\xi_{1}, \xi_{2}\right)}{d M_{12}}(s, t) M_{1}(d s)\right\} \xi_{2}(d t) \\
& +\int_{R}\left\{\int_{R}\left\{\int_{R} \frac{d\left(x, \xi_{1}\right)}{d M_{1}}(s) \frac{d\left(\xi_{1}, \xi_{2}\right)}{d M_{12}}(s, t) M_{1}(d s)\right\} \frac{d\left(\xi_{2}, \xi_{1}\right)}{d M_{21}}(t, u) M_{2}(d t)\right\} \xi_{1}(d u) \\
& +\int_{R}\left\{\int_{R}\left\{\int_{R} \frac{d\left(x, \xi_{1}\right)}{d M_{2}}(s) \frac{d\left(\xi_{2}, \xi_{1}\right)}{d M_{21}}(s, t) M_{2}(d s)\right\} \frac{d\left(\xi_{1}, \xi_{2}\right)}{d M_{12}}(t, u) M_{1}(d t)\right\} \xi_{2}(d u) \\
& -\cdots
\end{aligned}
$$

4. Application of the alternating projections theorem to bivariate stationary stochastic processes. Let $\mathbf{x}(t)=\left(x_{i}(t)\right)_{i=1}^{q} \in \mathscr{H}^{q}$ be a $q$-variate, stationary SP with the shift group $\left(U_{t}, t \in R\right)$. Here $\mathscr{H}^{q}$ is the cartesian product of a complex Hilbert space $\mathscr{H}$ with itself $q$-times, and $\left(U_{t}, t \in R\right)$ is a strongly continuous group of unitary operators on $\mathscr{H}$ such that $x_{i}(t+s)=U_{t} x_{i}(s)$ for all $s, t \in R(1 \leqq i \leqq q)$. By Stone's Theorem (cf. [8, p. 383]), there exists a spectral measure $E$ on the family $\mathscr{B}$ of Borel subsets of the real line $R$ such that $U_{t}=\int_{-\infty}^{\infty} e^{-i t \lambda} E(d \lambda)$. The $q \times q$ nonnegative, hermitian matrix-valued function $\mathbf{F}(\lambda)=2 \pi(\mathbf{E}(-\infty, \lambda] \mathbf{x}(0), \mathbf{x}(0))$ is called the spectral distribution of the SP. If $\mathbf{F}$ is absolutely continuous with respect to the Lebesgue measure (Leb), its derivative $\mathbf{F}^{\prime}$ is called the spectral density of the SP. 
Let $\mathscr{M}(t)$ be the past-present subspace of the SP $(\mathbf{x}(t), t \in R)$ up to time $t$ in $\mathscr{H}^{q}$, i.e., $\mathscr{M}(t)=\subseteq\{\mathbf{x}(s), s \leqq t\}$. Let $\mathscr{M}(-\infty)$ be its remote past subspace in $\mathscr{H}^{q}$, i.e., $\mathscr{M}(-\infty)=\bigcap_{t \leqq 0} \mathscr{M}(t)$.

An important result is that if $\mathscr{M}(-\infty)=\{0\}$, then $\mathbf{F}$ is a.c. with respect to Leb, and $\mathbf{x}(t)=\int_{-\infty}^{t} \mathbf{C}(t-s) \xi(d s)$, where $\mathbf{C}(\cdot)$ and $\mathbf{F}^{\prime}(\cdot)$ are related by a matrix-valued function $\Phi$ on $R$ such that

$$
\boldsymbol{\Phi}(\lambda) \boldsymbol{\Phi}^{*}(\lambda)=\mathbf{F}^{\prime}(\lambda) \text { a.e. } \lambda \text { and } \boldsymbol{\Phi}(\lambda)=\int_{0}^{\infty} \mathbf{C}(t) e^{i \lambda t} d t \in \mathbf{L}_{2}(R, \mathscr{B}, \text { Leb }),
$$

and if $\boldsymbol{\Phi}^{+}$denotes the holomorphic extension of $\boldsymbol{\Phi}$ to the upper half-plane, $\boldsymbol{\Phi}^{+}(i)$ is nonnegative hermitian, and

$$
\operatorname{det} \boldsymbol{\Phi}^{+}(i)=\exp \frac{1}{2 \pi} \int_{-\infty}^{\infty} \log \operatorname{det} \mathbf{F}^{\prime}(\lambda) \frac{d \lambda}{1+\lambda^{2}} .
$$

The function $\boldsymbol{\Phi}$ is called the generating function of the SP. It is not hard to show (cf. [5, p. 1372] and [11, p. 33]) that for each bounded interval $(a, b]$

$$
\xi(a, b]=2^{-1 / 2}\left\{\mathbf{h}(b)-\mathbf{h}(a)+\int_{a}^{b} \mathbf{h}(s) d s\right\}
$$

where $(\mathbf{h}(t), t \in R)$ is the weakly Markovian SP which is associated with the SP $(\mathbf{x}(t), t \in R)$.

We will need the following theorem, the proof of which is omitted and may be found in [11].

THEOREM 4.1. Let $(\mathbf{x}(t), t \in R)$ be a q-variate, stationary SP with spectral distribution $\mathbf{F}$. Then (a) $(\mathbf{x}(t), t \in R)$ is purely nondeterministic, i.e., $\mathscr{M}(-\infty)=\{0\}$ iff $\mathbf{F}$ is absolutely continuous with respect to the Lebesgue measure. If (a) holds, then

(b) $\subseteq\{\xi(a, b],-s<a \leqq b<t\}=\subseteq\{\mathbf{x}(u): s<u<t\}$.

(c) $\xi$ can be uniquely extended to the family $\mathscr{B}$ of Borel subsets of the real line $R$. If the $\mathrm{SP}$ is of rank $q$, or equivalently $\log \operatorname{det} \mathbf{F}^{\prime}(\lambda) /\left(1+\lambda^{2}\right) \in L_{1}(R, \mathscr{B}$, Leb), then for each Borel set $B$ of finite Lebesgue measure this extension has the representation

$$
\xi(B)=(2 \pi)^{1 / 2} \int_{-\infty}^{\infty} \hat{\chi}_{B}(\lambda) \Phi^{-1}(\lambda) \mathbf{E}(d \lambda) \mathbf{x}(0),
$$

where $\hat{\chi}_{B}(\lambda)=(2 \pi)^{-1 / 2} \int_{B} e^{-i \lambda t} d t$.

(d) The extension $\xi$ is an o.s. $\mathscr{H}^{q}$-valued measure over $(R, \mathscr{B})$, whose associated spectral measure is $\operatorname{Leb}(\cdot) \mathbf{I}_{q}$, where $I_{q}$ is the identity matrix of order $q$.

Definition 4.2. $\xi$ as defined in 4.1(c) is called the canonical measure of the SP $(\mathbf{x}(t), t \in R)$.

Now let $(\mathbf{x}(t), t \in R)$ be a bivariate, purely nondeterministic SP. Let $\mathbf{T}$ be the projection operator on $\mathscr{H}^{2}$ onto the subspace $\mathscr{M}(0)$, and $P$ be the projection operator on $\mathscr{H}$ onto the subspace $S\left\{\mathscr{M}_{1}(0)+\mathscr{M}_{2}(0)\right\}$, where $\mathscr{M}_{1}(0)$ and $\mathscr{M}_{2}(0)$ are the past-present subspaces of the component processes $x_{1}(t)$ and $x_{2}(t)$. It is easy to see (cf. [12, I, p. 131]) that the components of $\operatorname{Tx}(t)$ are precisely $P x_{1}(t)$ and 
$P x_{2}(t)$. Applying the results of $\S 3$ we will be able to obtain convergent infinite series expressions for $P x_{1}(t)$ and $P x_{2}(t)$, and hence the best linear prediction $\mathbf{T x}(t)$ is determinable.

Since $(\mathbf{x}(t), t \in R)$ is purely nondeterministic, so are its component processes $\left(x_{1}(t), t \in R\right)$ and $\left(x_{2}(t), t \in R\right)$ (cf. [11, p. 79]). Accordingly $\xi_{1}$ and $\xi_{2}$ will denote the canonical measures of the processes $\left(x_{1}(t), t \in R\right)$ and $\left(x_{2}(t), t \in R\right)$. Also $\mathscr{M}_{1}(t)$ and $\mathscr{M}_{2}(t)$ will denote the past-present subspaces of $\left(x_{1}(t), t \in R\right)$ and $\left(x_{2}(t), t \in R\right)$ up to time $t$ in $\mathscr{H}$.

We will be able to effect our algorithm under the following assumption.

Assumption 4.3. The spectral density $\mathbf{F}^{\prime}=\left[F_{i j}^{\prime}\right], 1 \leqq i, j \leqq 2$, of the bivariate, purely nondeterministic $\mathrm{SP}(\mathbf{x}(t), t \in R)$ satisfies the condition

$$
F_{12}^{\prime} /\left(F_{11}^{\prime}\right)^{1 / 2}\left(F_{22}^{\prime}\right)^{1 / 2} \in L_{1}(R, \mathscr{B}, \text { Leb })\left({ }^{4}\right) \text {. }
$$

REMARK 4.4. We assert that the SP has rank 2. For if its rank were less than 2, then $F_{11}^{\prime} F_{22}^{\prime}-F_{12}^{\prime} F_{21}^{\prime}=\operatorname{det} F^{\prime}=0$ a.e. on $R$ (cf. $[11$, p. 36$\left.]\right)$. Hence $F_{12}^{\prime} /\left(F_{11}^{\prime}\right)^{1 / 2}\left(F_{22}^{\prime}\right)^{1 / 2}$ $=1 \notin L_{1}(R, \mathscr{B}, \mathrm{Leb})$ which contradicts Assumption 4.3.

The component processes $\left(x_{1}(t), t \in R\right)$ and $\left(x_{2}(t), t \in R\right)$ are purely nondeterministic, and hence have the generating function $\phi_{1}, \phi_{2}$. We also note that since $\operatorname{det} F^{\prime} \geqq 0, F^{\prime} /\left(F_{11}^{\prime}\right)^{1 / 2}\left(F_{22}^{\prime}\right)^{1 / 2} \leqq 1$. Hence by Assumption 4.3,

$$
F_{12}^{\prime} /\left(F_{11}^{\prime}\right)^{1 / 2}\left(F_{22}^{\prime}\right)^{1 / 2} \in L_{\delta}(R, \mathscr{B}, \text { Leb }), \quad 1 \leqq \delta \leqq \infty .
$$

TheOREM 4.5. Let (i) the SP $(\mathbf{x}(t), t \in R)$ satisfy Assumption 4.3.

(ii) $\xi_{i}(i=1,2)$ be the canonical measure of the component process $\left(x_{i}(t), t \in R\right)$ $(i=1,2)$. Then Assumption 3.5 is satisfied, i.e., there exist Borel measurable functions $\varphi_{12}$ and $\varphi_{21}$ on $R^{2}$ such that

$$
\begin{aligned}
& \varphi_{i j}(\cdot, t) \in L_{2}(R, \mathscr{B}, \text { Leb) a.e. } t, \\
& \text { ess. lub. }\left|\varphi_{i j}(\cdot, t)\right|_{2, \text { Leb }}<\infty .
\end{aligned}
$$

(2) For each $B \in \mathscr{B}_{i j}, \chi_{B} \varphi_{i j} \in L_{1}\left(R^{2}, \mathscr{B} \times \mathscr{B}\right.$, Leb $\times$ Leb $)$.

(3) For each $B \times C \in \mathscr{B}_{i} \times \mathscr{B}_{j}$,

In fact if

$$
\left(\xi_{i}(B), \xi_{j}(C)\right)=\iint_{B \times C} \varphi_{i j}(s, t) d s d t .
$$

$$
\gamma_{i j}(t)=\frac{1}{2 \pi} \int_{-\infty}^{\infty} e^{-i t \lambda} \frac{F_{i j}^{\prime}(\lambda)}{\phi_{i}(\lambda) \phi_{j}(\lambda)} d \lambda\left({ }^{5}\right),
$$

then

$$
\varphi_{i j}(s, t)=\gamma_{i j}(s-t),
$$

where $\phi_{i}(i=1,2)$ is the generating function of the component process $\left(x_{i}(t), t \in R\right)$ $(i=1,2)$.

( $\left.{ }^{4}\right)$ By Cramér's Theorem (cf. [2, p. 221]) it is easy to see that this assumption is not void.

$\left.{ }^{5}\right)$ Since $\left|\phi_{i}\right|^{2}=F_{i i}^{\prime}(i=1,2)$, by $4.4, F_{i j}^{\prime} / \phi_{i} \phi_{j}$ is in $L_{\delta}(R, \mathscr{B}$, Leb $), 1 \leqq \delta \leqq \infty$. 
Proof. Let $\gamma_{i j}(t)=(1 / 2 \pi) \int_{-\infty}^{\infty} e^{-i t \lambda}\left(F_{i j}^{\prime}(\lambda) / \phi_{i}(\lambda) \phi_{j}(\lambda)\right) d \lambda$. We define $\varphi_{i j}$ on $R^{2}$ by

$$
\varphi_{i j}(s, t)=\gamma_{i j}(s-t) \text {. }
$$

Since $\left|\phi_{i}\right|^{2}=F_{i t}^{\prime}$, by (i) and $4.4, F_{i j}^{\prime} / \phi_{i} \phi_{j} \in L_{2}(R, \mathscr{B}$, Leb). Hence by Plancherel's Theorem we have $\int_{-\infty}^{\infty}\left|\varphi_{i j}(s, t)\right|^{2} d s=\int_{-\infty}^{\infty}\left|\gamma_{i j}(s-t)\right|^{2} d s=\left.(1 / 2 \pi)\left|F_{i j}^{\prime}\right| \phi_{i} \phi_{j}\right|_{2, \text { Leb }} ^{2}<\infty$, so that ess. lub.teR $\left|\varphi_{i j}(\cdot, t)\right|_{2, \text { Leb }}=\left.(1 / 2 \pi)\left|F_{i j}^{\prime}\right| \phi_{i} \phi_{j}\right|_{2, \text { Leb }}<\infty$. Hence (1) is proved.

Also by (i) and 4.4, $\varphi_{i j} \in L_{\infty}\left(R^{2}, \mathscr{B} \times \mathscr{B}\right.$, Leb $\times$ Leb) and hence (2) is satisfied.

By 4.1 , for each $B \in \mathscr{B}_{i}(i=1,2)$ we have

$$
\xi_{i}(B)=(2 \pi)^{1 / 2} \int_{-\infty}^{\infty} \hat{\chi}_{B}(\lambda) \phi_{i}^{-1}(\lambda) E(d \lambda) x(0) \quad(i=1,2) .
$$

From (i) it follows that $F_{i j}^{\prime} \mid \phi_{i} \phi_{j} \in L_{1}(R, \mathscr{B}, \mathrm{Leb})$, so that by Fubini's Theorem for each $B \times C \in \mathscr{B}_{i} \times \mathscr{B}_{j}$,

$$
\begin{aligned}
\left(\xi_{i}(B), \xi_{j}(C)\right) & =2 \pi\left(\int_{-\infty}^{\infty} \hat{\chi}_{B}(\lambda) \phi_{i}^{-1}(\lambda) E(d \lambda) x_{i}(0), \int_{-\infty}^{\infty} \hat{\chi}_{C}(\lambda) \phi_{j}^{-1}(\lambda) E(d \lambda) x_{i}(0)\right) \\
& =2 \pi \int_{-\infty}^{\infty} \hat{\chi}_{B}(\lambda) \overline{\hat{\chi}_{C}(\lambda)} \phi_{i}^{-1}(\lambda) \overline{\phi_{j}^{-1}(\lambda)}\left(E(d \lambda) x_{i}(0), x_{j}(0)\right) \\
& =\int_{-\infty}^{\infty} \hat{\chi}_{B}(\lambda) \overline{\hat{\chi}_{C}(\lambda)} \cdot \frac{F_{i j}^{\prime}(\lambda)}{\phi_{i}(\lambda) \phi_{j}(\lambda)} d \lambda \quad \text { (cf. [11, p. 82]) } \\
& =\frac{1}{2 \pi} \iint_{B \times C}\left\{\int_{-\infty}^{\infty} e^{-i(s-t) \lambda} \frac{F_{i j}^{\prime}(\lambda)}{\phi_{i}(\lambda) \phi_{j}(\lambda)} d \lambda\right\} d s d t \\
& =\iint_{B \times C} \gamma_{i j}(s, t) d s d t .
\end{aligned}
$$

We now state the main theorem of this section.

MAIN THeOREM 4.6. Let (i) the bivariate SP $(\mathbf{x}(t), t \in R)$ satisfy Assumption 4.3.

(ii) $\mathscr{M}_{i}(0)(i=1,2)$ be the past-present subspace of the component process $\left(x_{i}(t), t \in R\right)(i=1,2)$, and let $P_{1}, P_{2}$, and $P$ be the projection operators on $\mathscr{H}\left({ }^{6}\right)$ onto the subspaces $\mathscr{M}_{1}(0), \mathscr{M}_{2}(0)$, and $\subseteq\left\{\mathscr{M}_{1}(0)+\mathscr{M}_{2}(0)\right\}$.

(iii) $\phi_{i}(\lambda)=\int_{0}^{\infty} e^{i \lambda t} c_{i}(t) d t$,

$$
\gamma_{i j}(t)=\frac{1}{2 \pi} \int_{-\infty}^{\infty} e^{-i t \lambda} \frac{F_{i j}^{\prime}(\lambda)}{\phi_{i}(\lambda) \phi_{j}(\lambda)} d s,
$$

where $\phi_{i}(i=1,2)$ is the generating function of the component process $\left(x_{i}(t), t \in R\right)$, $1 \leqq i \leqq 2$.

(iv) $\xi_{i}(i=1,2)$ be the canonical measure of the component process $\left(x_{i}(t), t \in R\right)$ $(i=1,2)$.

$\left(^{6}\right) \mathscr{H}$ is a Hilbert space such that $x_{i}(t) \in \mathscr{H}$ for all $t \in R, i=1,2$. 
Then for each $\tau \geqq 0$,

$$
\begin{aligned}
\operatorname{Px}_{i}(\tau)= & \int_{0}^{\infty} c_{i}(\tau+s) \xi_{i}(-d s)+\int_{0}^{\infty}\left\{\int_{-\tau}^{0} c_{i}(\tau+s) \gamma_{i j}(t-s) d s\right\} \xi_{j}(-d s) \\
& -\int_{0}^{\infty}\left\{\int_{0}^{\infty}\left\{\int_{-\tau}^{0} c_{i}(\tau+s) \gamma_{i j}(t-s) d s\right\} \gamma_{j i}(u-t) d t\right\} \xi_{i}(-d u)+\cdots
\end{aligned}
$$

Proof. By the third paragraph of $\$ 4$ and $4.1(b)$,

(1)

$$
\begin{aligned}
x_{i}(\tau) & =\int_{-\tau}^{\infty} c_{i}(\tau+s) \xi_{i}(-d s), \\
\mathscr{M}_{i}(0) & =\subseteq\left\{\xi_{i}(B), B \subseteq(-\infty, 0] \text { and } B \in \mathscr{B}_{i}\right\} .
\end{aligned}
$$

By $4.5(\mathrm{c}),\left(d\left(\xi_{i}, \xi_{j}\right) / d M_{i j}\right)(s, t)=\gamma_{i j}(s-t)$ and Assumption 3.5 is satisfied. Hence by (i) and 3.9, we have

$$
\begin{aligned}
P_{i} x_{i}(\tau) & =\int_{0}^{\infty} c_{i}(\tau+s) \xi_{i}(-d s), \\
P_{j} x_{i}(\tau) & =\int_{0}^{\infty}\left\{\int_{-\tau}^{\infty} c_{i}(\tau+s) \gamma_{i j}(t-s) d s\right\} \xi_{j}(-d t), \\
P_{i} P_{j} x_{i}(\tau) & =\int_{0}^{\infty}\left\{\int_{0}^{\infty}\left\{\int_{-\tau}^{\infty} c_{i}(\tau+s) \gamma_{i j}(t-s) d s\right\} \gamma_{j i}(u-t)\right\} \xi_{i}(-d u), \\
P_{j} P_{i} x_{j}(\tau) & =\int_{0}^{\infty}\left\{\int_{0}^{\infty} c_{i}(\tau+s) \gamma_{i j}(t-s) d s\right\} \xi_{j}(-d t) .
\end{aligned}
$$

Hence, after some simplification, we have the result by 3.10. (Q.E.D.)

The following is an immediate corollary to this theorem.

COROLlaRY 4.7. With the notation of 4.6 , if $Q$ is the projection operator on $\mathscr{H}$ onto the orthogonal complement of $\Im\left\{\mathscr{M}_{1}(0)+\mathscr{M}_{2}(0)\right\}$ in $\mathscr{H}$ then for each $\tau \geqq 0$

$$
\begin{aligned}
Q x_{i}(\tau)= & \int_{-\tau}^{0} c_{i}(\tau+s) \xi_{i}(d s)-\int_{0}^{\infty}\left\{\int_{-\tau}^{0} c_{i}(\tau+s) \gamma_{i j}(t-s) d s\right\} \xi_{j}(-d t) \\
& +\int_{0}^{\infty}\left\{\int_{0}^{\infty}\left\{\int_{-\tau}^{0} c_{i}(t+s) \gamma_{i j}(t-s) d s\right\} \gamma_{j i}(u-t) d t\right\} \xi_{i}(-d u)-\cdots
\end{aligned}
$$

Acknowledgement. The author wishes to thank Professor P. R. Masani for suggesting the problems discussed in this paper and for his valuable comments while the author was at Indiana University.

\section{BIBLIOGRAPHY}

1. A. S. Besicovitch, A general form of the covering principle and relative differentiation of additive functions. II, Proc. Cambridge Philos. Soc. 42 (1946), 1-10.

2. H. Cramér, On the theory of stationary random processes, Ann. of Math. 41 (1940), 215230.

3. P. R. Halmos, Measure theory, Van Nostrand, Princeton, N. J., 1950. 
4. L. H. Koopmans, On the coefficient of coherence for weakly stationary stochastic processes, Ann. Math. Statist. 35 (1964), 532-549.

5. P. Masani and J. Robertson, The time domain analysis of a continuous weakly stationary stochastic process, Pacific J. Math. 11 (1962), 1361-1378.

6. R. F. Matveev, On multi-dimensional regular stationary processes, Theor. Probability Appl. 6 (1961), 149-165.

7. J. von Neumann, Functional operators, Vol. II, Annals of Mathematics Studies No. 21, Princeton Univ. Press, Princeton, N. J., 1950.

8. F. Riesz and B. Sz.-Nagy, Functional analysis, Ungar, New York, 1955.

9. M. Rosenberg, The square-integrability of matrix-valued functions with respect to a nonnegative hermitian measure, Duke Math. J. 31 (1964), 291-298.

10. H. Salehi, The Hilbert space of square-integrable matrix-valued functions with respect to a o-finite nonnegative, hermitian measure and stochastic integrals, Research memorandum, Michigan State Univ., East Lansing, 1966.

11. - The prediction theory of multivariate stochastic processes with continuous time, Doctoral Thesis, Indiana Univ., Bloomington, 1965.

12. N. Wiener and P. Masani, The prediction theory of multivariate stochastic processes. I, Acta Math. 98 (1957), 111-150; II, Acta Math. 99 (1958), 93-137.

Michigan State University,

East Lansing, Michigan 